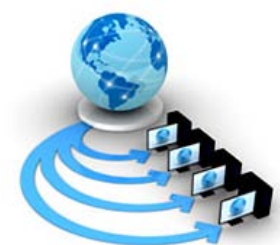

Volume 9, No. 2, March-April 2018

\title{
AN EXPLORATION TO IMAGE RECONSTRUCTION APPROACHES TO REPAIR REAL TIME IMAGES
}

\author{
Nishant Jakhar \\ Ph.D. Scholar \\ University institute of engineering and technology \\ Maharshi Dayanand University Rohtak, India
}

\author{
Rainu Nandal \\ Assistant professor \\ University institute of engineering and technology \\ Maharshi Dayanand University Rohtak, India
}

\begin{abstract}
When the image is captured from the source using some scanner or the camera, it can have number of deficiencies because of the camera, environment or lack of expertise problems. These problems results distorted capturing of images. But to perform any image processing operation, it is required to resolve these impurities over the image. In this paper, the importance of image restoration is defined against various problems of images. The paper has discussed some of effective image reconstruction methodologies.
\end{abstract}

Keywords: Image Reconstruction, Noise, Impurities, Pixel Shift

\section{INTRODUCTION}

Image Processing comes under signal processing for getting the input image such as some photograph or the video frame. The output of the processing activity is also in same image or the video frame form. The processing over the images is performed under the characteristics analysis or parameter based analysis performed over the image. There are number of evolving image processing techniques that accept the images in the form of two dimensional signals and apply different signal processing operation on it. Image processing can be performed in different associated forms such as signal processing, optical or analog processing etc. These all image processing activities begin after the taken the image as input. This image acquisition process is defined under the physical processing that converts the input signal form of image to physical image[1][2][3].

The signal image can be available either in digital or analog form. The output of the image is identified as the characteristics identification and exploration for the image. There is different kind of image processing activities associated with it. During this acquisition process, the capturing of image is done through the camera and converted to physical form by defining the image format or the type of the photograph. The transition of the image from one format to other can be done using some photographic software. This software type is included with the actual image form and performed the actual acquisition of the image[4][5].

These image forms of images are defined along with the specification of colors, shading and the capturing of the image under the transition of image form one form to other. These image forms are defined under the photography specification along with burning of image in the form of film by performing the chemical reaction and to generate the actual image. To generate this image, the chemical reaction is exposed to light. This image transition process is performed in a dark room. This kind of processing of image acquisition is not used these days. This kind of activity requires the efforts and the special training to the convert the image from the films. But today more effective sources are available to perform the image acquisition by using the digital cameras. There are number of processing applications and tools that are engaged with digital form. These applications include the gesture recognition, medical image processing, remote sensing etc. There are some specialized computer programs that are used to improve the image quality and to correct the defects over the images. These kinds of programs are defined as the algorithmic approaches to process on actual data and to reduce the image distortion and signal distortion. There are number of associated approaches to obtain the improved image and to add the light to the images[7][8][9]. The problems in noise are listed in this section.

\section{A) Image Noise}

Image noise is the distributed noise particles over the images that affects the image brightness and color information at random position with random intensity. This kind of noise occurs as the image is captured from the cameras or the scanner. The problem in the circuitry or in the sensor affects the actual pixel intensity of the images and distorts the image at some points. The noise over the image is considered as the undesirable particles that destroy the actual image information completely or partially. The unwanted fluctuation over the image signal value is also considered as the noise vector to the image. This kind of noise is considered as the dithering particles that affects the quality of the image[10][11].

The desirable quality of the image is considered as the changes performed over the image intensity or the brightness. There are number of factors associated with image processing that performs the variation over the image in terms of brightness or the color value of the image. The variation in this noise type is generally random in terms of position as well as the intensity value added over the pixel points. The significant change is performed over the image object is about to improve the image quality for low contrast images. 
B)

Sharpening

Sharpening is considered as the effective transformation activity that affects the image quality and retrieves the image feature so that the improvement to the image will be done. The extraction to the image edges is done using this operation. The edges of the images are considered as the important feature that adds the visual effect to the image so that the image features will be improved. The improvement to the image will be done so that the blurred version of the image will be ignored and better image representation will be done. But when the feature distribution over the image is not equalizing, it is considered as the problem to the image. In this form, the pixel is brighter then the lighter image areas over the image. The result is here been taken to increase the contrast over the image and between the image pixels and the neighbor values. The nature of the image sharpening is here defined as the blurring radius and the difference analysis is performed between the actual pixel value and the neighbor pixels[12][13].

\section{C) Image Restoration}

Image restoration is about to improve the image feature by retouching the image and by retrieving the image feature back so that the effective result image will be generated. These all image forms can be generated from the actual photography and requirement of the printing. The image editing to the image can be done under the content specification and creativity maintenance.

There are number associated sources that specializes the image editing the restoration of the images under the specification of retouching of images under different industry level process analysis and variation analysis. These all kind of industries associated with the images are Travel, Hotel, Real Estate etc.

The registration of images is actually done to compensate the defects of these images and to degrade the image features so that misfocus problem over the image will be rectified. There are number associated cases that perform the good estimation over the images and perform the undo operation to the actual blur operation so that the image restoration will be done. The image restoration is actually applied to enhance the image when the image features are not completely available as well as the image features are distorted. These are number of such associated problems that can be rectified over the images[14][15].

In this paper, a study to different image restoration approaches is discussed. In this section, the exploration to the image processing and various issues associated with image processing are discussed. The paper has also discussed image restoration in brief. In section II, the work defined by earlier researchers is discussed. In section III, some of the effective image restoration approaches are presented. In section IV, the conclusion obtained from the work is presented.

\section{EXISTING WORK}

Lot of work is already presented by different researchers on image restoration, denoising and image fusion. Some of the contributions of earlier researchers are discussed in this section. R. Maruthi[1] has presented a work on information level analysis under different parameters. Based on these parameters the reconstruction of images is done. Author has considered visibility parameters and frequency driven parameters for the comparison applied on different blocks on images. Based on this block level analysis effective formation of result image is done. Author has defined a pixel selection approach under magnitude analysis to measure the activity of information block and applied image reconstruction on it. Author reduces the chances on any kind of data loss during the image reformation process. Shuo-Li Hsu[2] defined a region driven approach applied on visual features so that the reliable and accurate formation of input image will be done. Author has formed the new image by defining the visual feature analysis so that the new segmented image will be formed. Based on this analysis, the bad region will be identified which region level repairing is performed. This repairing is performed by using the mathematical operators along with pixel shift method to identify the most suitable pixel in specific area. Author defined the region repairing under adaptive capacity analysis and determination so that reconstruction of image will be done.

ManjushaDeshmukh[3] presented an image quality analysis approach applied on muti focus images. Author used the PCA formation to perform wavelet decomposition on images at the early step and later on the sub image based reformulation of images will be done. Author applied the image decomposition and image reconstruction so that the new image will be composed from sub images. Author defined the random space analysis approach for reconstruction of image and to improve the quality of image under different parameters. Author defined the restoration process and applied the image reconstruction under plentiful image formation and reconstruction. MiladGhantous[4] has defined an object extraction image formation approach using restoration method. Author defined the feature analysis approach applied at image blocks to repair the image and to identify the gradient change at the grayscale image. Author defined two levels approach in which at first stage actual object part is identified and later on the reconstruction of object will be formed based on pixel selection approach. Tao Wan[5] defined a work to generate a new formed image by performing the low level image repairing at compressed image. Author applied the pattern analysis on this image to drive the sample based on acceptability and compressive formation can be applied. Author also defined the compressive vector based sample selection so that the new image block will be formed without any impurity over the block. As these kinds of blocks are collected the final image is constructed.

AndrejaSvab[6] defined a work on image restoration on high resolution image to apply the spatial analysis and generate the effective spectral change. Author has defined adaptive reconstruction approach so that the multiple blocks will be analyzed parallel and the effective reconstruction of image will be done. Author has defined the image formation under spectral analysis so that reliable information can be obtained. SaurabhSingh[7] has presented reconstruction process for biometric face images. Author deal with the challenges in facial recognition by forming the eigen value analysis. Author identifies the occlusion block over the facial part and 
performs reconstruction of image. This image formation is done under different experimentation methods so that reliable and accurate reformation of image will be done. Hong Zheng[8] defined decomposition approach for image reformation based on spatial feature analysis. Author defined multi level pixel formation so that new pixel will be generated to repair the image effectively. G. Simone[9] has presented a work to discuss and resolve the challenges of image acquisition and provide reliable image formation based on the pre analysis on the image. Author defined the decomposition process applied on object model to provide the quality analysis and to form a new image out of the valid image specification. Author defined the object level formation and defined the restoration process applied on multiple images. WenzhongShi[10] defined the DWT effective approach for generating a new image by performing feature level analysis on it. This image formation is based on the statistical features so that new image blocks will be obtained. Author defined the decision vectors for repairing of image under effective feature generation.

Hou et al.[16] used the blur kernel estimation method to suppress the salt-and-pepper noise. The method has conducted the joint estimation to image quality and reconstructs the image to remove the noise elements. He et al.[16] provided a cross scale and cross RGB channel based method to denoise the image. The unified model is defined to conduct the interpolation for improving the quality of noise corrupted images. Sudeesh et al.[18] has provided an analytical study on different filtration methods to remove the noise from medical images. The region segmentation method was integrated to preserve the critical region from the image. Author first estimated the noise and later applied the selective weighted median filter to rectify the critical noisy regions. Rajesh et al.[19] has provided the decision tree based method to identify the corrupted region within the image. The edge region was preserved by this method to reconstruct the image. The mathematical computation method was applied to retain the visual quality and to improve the performance of image rectification.

\section{IMAGE RESTORATION METHODOLOGIES}

The paper already has discussed the issues associated with image processing in the form of noise, sharpen etc. Some of other problem includes color balancing, contrast unbalancing etc. There are number of approaches presented by earlier researchers to resolve these problems. In this paper some of these approaches are discussed. These approaches include repairing as well as enhancing the image features.

\section{A) Histogram Equalization}

Histogram is taken as the effective processing activity that is used to perform the image enhancement under the method specification. This kind of process is performed under multiple passes. This technique is defined under the histogram specification and pixel variation analysis so that the pixel level differencing and the alteration will be done effectively. The histogram is here considered to locate the block over the image and to analyze the color and intensity analysis as the effective measure to the system.
There is the requirement of some refinement approach that can be applied recursively under the specification of histogram and generating the blocks so that the image can be divided in smaller segments. These repeated smaller segments are formed to generate the effective blocks[2]. The main disadvantage of this method is to identify the significant peak value and valley value so that the effective decisions will be taken.

\section{B) Mean Shift Method}

This is considered as the effective reconstruction approach defined to search the local maximum density point specification and group generation under the density point specification. There are number of reconstruction approaches under the pixel specification. The approach can be applied for reformation process for each pixel of image. The density analysis for repairing process is defined in equation (1)

$$
\hat{f}(x)=\frac{c}{n h^{d}} \sum_{i=1}^{n} K\left(\left\|\frac{x-x_{i}}{h}\right\|\right)^{2}
$$

Here, $h$ is bandwidth d represents dimensionality c constant value for normalization

K represents the kernel function for density estimation. This method uses the kernel parameter as the width vector to estimate the area density respective to specific point as well as specific to the particular intensity. The educational representation of this function for estimation of next update is given in equation (2)

$$
y_{j+1}=\frac{\sum_{i=1}^{n} x_{i} K\left(\left\|\frac{y_{j}-x_{i}}{h}\right\|^{2}\right)}{\sum_{i=1}^{n} K\left(\left\|\frac{y_{j}-x_{i}}{h}\right\|^{2}\right)}
$$

This estimation is respective to region and used to identify the local maximal point under the density level estimation. The estimation is here based on bandwidth, range bandwidth specification and the region specification.

\section{C) Morphological Operators}

Morphological Operators are defined as the set of mathematical operators that accept the input in matrix form and perform the geometrical structure based operation under set theory. This algorithm approach is the combination of set theory, lattices and the topological operations. Image processing is one of the major applied of morphological operator where the image or the image regions are considered as the input object. The geometric based operations are applied using morphological concept under the shape, size and connectivity analysis. This kind of categorization is based on continuous and discrete spaces. The morphological image processing is here defined under set of operators with the specification of associated characterization. 
A piece wise or region specific evaluations are conducted by spatial correlation model to restore the noisy image. The model performs the neighbor pixel analysis within the region to identify the degree of variation. The neighbor correlation evaluation provided by this method is shown in equation (3).

$$
\mathrm{y}(\mathrm{i}, \mathrm{j})=\sum_{p, q \in\{i, j\}^{n}} \alpha_{p, q} y(i+p, j+q)+n(i+j)
$$

Where, $y$ is the input image and $(i, j)$ represent the pixel position. $(p, q)$ represent the window size analyzed within the region for neighborhood pixel analysis. The semantic analysis on the image is performed to gain the spatial information for spatial structure and to reconstruct the image. The block specific scanning is also performed to achieve the selective rectification of partial unbalanced image.

\section{CONCLUSION}

In this paper, a study on different restoration processes is shown for real time images. The paper has discussed, the type of restoration under the feature vector analysis as well describe the challenging areas of image restoration. Later on the research methodology of some of the most effective restoration algorithms is defined in this paper.

\section{REFERENCES}

[1] R.Maruthi, Dr.K.Sankarasubramanian, "MULTI FOCUS IMAGE FUSION BASED ON THE INFORMATION LEVEL IN THE REGIONS OF THE IMAGES", Journal of Theoretical and Applied Information Technology, Vol 3 , Issue 4, pp 80-85, 2010

[2] Shuo-Li Hsu, Peng-Wei Gau, I-Lin Wu, and Jyh-Horng Jeng, "Region-Based Image Fusion with Artificial Neural Network", International Journal of Computer and Information Engineering, Vol 3, Issue 5, 2009

[3] Deshmukh, Manjusha, and Udhav Bhosale. "Image fusion and image quality assessment of fused images." International Journal of Image Processing (IJIP) 4, no. 5 (2010): 484.

[4] Ghantous, Milad, Soumik Ghosh, and Magdy Bayoumi. "A gradient-based hybrid image fusion scheme using object extraction." In Image Processing, 2008. ICIP 2008. 15th IEEE International Conference on, pp. 1300-1303. IEEE, 2008.

[5] Wan, Tao, Nishan Canagarajah, and Alin Achim. "Compressive image fusion." In Image Processing, 2008. ICIP 2008. 15th IEEE International Conference on, pp. 1308-1311. IEEE, 2008

[6] Andreja Svab Lenarcic and KrištofOštir, "High-resolution image fusion: Methods to preserve spectral and spatial resolution",Photogrammetric Engineering and Remote Sensing, Vol. 72, No. 5, pp. 565-572, May 2006
Singh, Saurabh, Aglika Gyaourova, George Bebis, and Ioannis Pavlidis. "Infrared and visible image fusion for face recognition." In Biometric Technology for Human Identification, vol. 5404, pp. 585-597. International Society for Optics and Photonics, 2004.

[8] Zheng, Hong, Dequan Zheng, Yanxiang Hu, and Sheng Li. "Study on the optimal parameters of image fusion based on wavelet transform." journal of computational information systems 6, no. 1 (2010): 131-137.

[9] Simone, Giovanni, Alfonso Farina, Francesco Carlo Morabito, Sebastiano B. Serpico, and Lorenzo Bruzzone. "Image fusion techniques for remote sensing applications." Information fusion 3, no. 1 (2002): 3-15.

[10] Shi, Wenzhong, ChangQing Zhu, Yan Tian, and Janet Nichol. "Wavelet-based image fusion and quality assessment." International Journal of Applied Earth Observation and Geoinformation 6, no. 3-4 (2005): 241251

[11] Alparone, Luciano, Luca Facheris, Stefano Baronti, Andrea Garzelli, and Filippo Nencini. "Fusion of multispectral and SAR images by intensity modulation." In Proceedings of the 7th International Conference on Information Fusion, pp. 637-643. 2004.

[12] Gemma Piella, "New quality measures for image fusion", 2004

[13] Singh, Richa, Mayank Vatsa, and Afzel Noore. "Integrated multilevel image fusion and match score fusion of visible and infrared face images for robust face recognition." Pattern Recognition 41, no. 3 (2008): 880893.

[14] Riyahi, R., C. Kleinn, and H. Fuchs. "Comparison of different image fusion techniques for individual tree crown identification using quickbird image." International Society for Photogrammetry and Remote Sensing, HighResolution Earth Imaging for Geospatial Information 38 (2009): 1-4.

[15] Hanif, Mohammad, and Usman Ali. "Optimized visual and thermal image fusion for efficient face recognition." In Information Fusion, 2006 9th International Conference on, pp. 1-6. IEEE, 2006.

[16] L. Hou, H. Liu, Z. Luo, Y. Zhou and T. K. Truong, "Image deblurring in the presence of salt-and-pepper noise," 2017 IEEE International Conference on Image Processing (ICIP), Beijing, China, 2017, pp. 2389-2393.

[17] Z. He, K. Tang and L. Fang, "Cross-scale color image restoration under high density Salt-and-Pepper Noise," 2017 IEEE International Conference on Image Processing (ICIP), Beijing, China, 2017, pp. 3780-3784.

[18] K. V. Sudheesh and L. Basavaraj, "Selective weights based median filtering approach for impulse noise removal of brain MRI images," 2016 International Conference on Electrical, Electronics, Communication, Computer and Optimization Techniques (ICEECCOT), Mysuru, 2016, pp. 60-65.

[19] R. Rajesh and P. Malathi, "An effective denoising and enhancement technique for removal of random impulse noise in images," 2016 IEEE International Conference on Advances in Electronics, Communication and Computer Technology (ICAECCT), Pune, 2016, pp. 256-261. 